\title{
Sistemas electrónicos modernos en la organización de webinars
}

\section{Modern electronic systems in the organization of training webinars}

\author{
Inna K. Kirillova \\ innes_05-81@mail.ru \\ Moscow State University of Civil Engineering, Moscow, Russia \\ https://orcid.org/0000-0002-9873-4832 \\ Yana V. Zubkova \\ yanazubkova@yandex.ru \\ Moscow State University of Civil Engineering, Moscow, Russia \\ https://orcid.org/0000-0002-2484-7316 \\ Julia M. Tsarapkina \\ julia_carapkina@mail.ru \\ Russian State Agrarian University - Moscow Timiryazev Agricultural Academy, Moscow, Russia \\ https://orcid.org/0000-0002-3807-4211 \\ Anzhella V. Ilyasova \\ strochno@mail.ru \\ Penza State Technological University, Penza, Russia \\ https://orcid.org/0000-0001-5495-1424 \\ Yulia S. Rubinchik \\ luch2118@mail.ru \\ Moscow Region State University, Mytishchi, Russia \\ https://orcid.org/0000-0001-6067-2835
}

Recibido: 03/12/2020

Aceptado: 14/01/2021

\section{Resumen}

El propósito del estudio fue investigar la experiencia del uso de sistemas electrónicos modernos en la organización de webinars realizados con fines educativos. La metodología aplicada para realizar el estudio, se organizó una encuesta, cuyos participantes eran estudiantes de tres escuelas superiores en Rusia. Como conclusión se presentan los datos obtenidos en el transcurso del estudio nos permiten hablar de un aumento en el número de webinars y la expansión de oportunidades de formación para estudiantes de instituciones de educación superior a través de la implementación de sistemas electrónicos modernos. 
Inna K. Kirillova, Yana V. Zubkova, Julia M. Tsarapkina, Anzhella V. Ilyasova, Yulia S. Rubinchik.

Palabras clave: sistemas electrónicos, webinar, educación profesional, proceso educativo, software.

\section{Abstract}

The purpose of the study was to investigate the experience of using modern electronic systems in the organization of webinars held for educational purposes. Methodology. To conduct the study, a survey was organized, the participants of which were students of three higher schools in Russia. As a conclusion, the data obtained in the course of the study are presented, allowing us to speak of an increase in the number of webinars and the expansion of training opportunities for students of higher education institutions through the implementation of modern electronic systems.

Key Words: electronic systems, webinar, professional education, educational process, software.

\section{Introducción}

La implementación de recursos electrónicos modernos en el espacio educativo actual es una condición necesaria para mantener y mejorar la calidad de la educación. La necesidad de resolver el problema del desarrollo de tecnologías digitales está indicada por el decreto del presidente de la Federación de Rusia núm. 204 de 7 de julio de 2018 (Decreto $\mathrm{N}^{\circ} 204,2018$ ) "sobre metas nacionales y objetivos estratégicos para el desarrollo de la Federación de Rusia para el período hasta 2024 "(Vaganova et al., 2020a). El programa nacional "Economía digital de la Federación de Rusia" revela la necesidad de capacitar al personal para trabajar en el entorno digital. Las instituciones educativas profesionales buscan e implementan herramientas electrónicas en el proceso educativo, cuyo uso proporciona una expansión de las oportunidades de formación para los estudiantes. La interacción remota de sujetos en el proceso educativo se lleva a cabo actualmente mediante múltiples programas que promueven la interacción virtual y la organización del proceso educativo. Entre ellos, puede seleccionar como Zoom, Mirapolis Virtual Room Viewer Time Viewer. Un producto de software, M-M Moodle es un sistema de aprendizaje a distancia abierto.

El propósito del estudio es estudiar la experiencia del uso de plataformas electrónicas modernas en la organización de webinars que se realizan con fines educativos (Shcerbakova \& Shcerbakova, 2019).

Los seminarios web de formación que proporcionan interacción de todos los participantes en el proceso pedagógico son un medio real de aprendizaje en línea (Eliseeva et al., 2020). Los webinars de formación permiten a los sujetos del proceso educativo intercambiar experiencias de forma activa, obtener información valiosa para la superación profesional continua y la autoeducación (Chil et al., 2020). La amplia gama funcional del webinar contribuye a la organización del proceso educativo a un alto nivel, contribuyendo a la formación de competencias de especialistas en 
condiciones remotas. El software moderno utilizado por las instituciones de educación superior permite a los estudiantes participar en reuniones virtuales independientemente de la ubicación del estudiante, lo que amplía las oportunidades de capacitación (Vaganova et al., 2020b).

Para cumplir con los requisitos de la legislación y la calidad de la formación de competencias profesionales en condiciones remotas, es necesario analizar la implementación de las capacidades de los sistemas electrónicos para organizar la formación.

\section{Marco teórico}

Los sistemas de aprendizaje a distancia Zoom, Mirapolis Virtual Room o Time Viewer le permiten crear audiencias virtuales que reúnen a todos los participantes del webinar. El webinar requiere una alta accesibilidad para los estudiantes; Ahorro de tiempo para la organización de clases. La característica principal de un webinar es su interactividad.

Un webinar es un tipo de conferencia web en la que cada participante se comunica a través de Internet. El propósito de los seminarios web de capacitación es una introducción a los problemas del área temática. Para participar en el seminario web, necesita una cámara web incorporada en su computadora portátil o una externa conectada a su computadora a través de USB (Vaganova et al., 2020c).

El sistema de aprendizaje a distancia Mirapolis Virtual Room es un producto relevante para la organización de webinars de formación (Pichugina \& Bondarchuk, 2019). Este software le permite realizar reuniones en línea en diferentes formatos. Los usuarios participan en ellos independientemente de su ubicación. No es necesario instalar software adicional (Vaganova et al., 2019a). Las características de Mirapolis incluyen: conferencias de audio y video; ver varios documentos y materiales multimedia de todos los participantes; organización del trabajo conjunto en un tablero electrónico con una amplia gama de herramientas; interacción de los participantes a través de un chat común; realizar encuestas; grabación de eventos; intercambio de archivos (Vaganova et al., 2019b). El uso de la Sala Virtual Mirapolis brinda la apertura informativa de una institución educativa profesional.

Team Viewer ofrece opciones flexibles para compartir archivos. En otras palabras, es posible transferir archivos de cualquier tamaño durante el seminario web. Además, los sujetos del proceso educativo pueden crear grupos de canales.

Zoom es una plataforma basada en la nube para videoconferencias, seminarios web y otros eventos en línea. Zoom tiene una amplia gama de herramientas para la colaboración. La pizarra le permite trabajar en conjunto para crear notas, comentarios, ediciones y otros comentarios. Es posible organizar charlas colectivas. Zoom chat le permite organiz ar grupos privados o abiertos, crear encuestas y compartir contenido 
educativo. Puede haber varios chats dentro de un solo proyecto (Nagovitsyn et al., 2020). Las videoconferencias en Zoom se inician utilizando iCal, Gmail, Outlook y otras herramientas populares (Nikonova et al., 2019b). La plataforma proporciona capacidades integradas de audio y video HD. Las conferencias pueden contar con un máximo de 1000 personas. Se pueden mostrar hasta 49 participantes en la pantalla. Las conferencias se graban en la nube o en un dispositivo de almacenamiento local (Ponachugin, 2020). Puede ver grabaciones y tomar notas sobre ellas. Debido a la gran cantidad de usuarios, la carga en el sistema está creciendo, pero Zoom puede manejar cientos de miles de conferencias simultáneamente.

Esta no es una lista exhaustiva de herramientas electrónicas utilizadas por las instituciones de educación superior durante la transición masiva a la educación a distancia. Los servicios de intercambio de archivos como Dropbox, Skype, WhatsApp, FaceTime y otros tienen una gran demanda (Nikonova et al., 2019a). La organización de sesiones de capacitación en modo de videoconferencia incluye informar obligatoriamente a los oyentes sobre el webinar, verificaciones preliminares de comunicación y proporcionar un hipervínculo a la dirección del recurso en la red (URL). El mensaje informativo contiene el nombre del webinar, información sobre el anfitrión, el inicio y la duración, metas y objetivos, e información sobre la estructura y el contenido.

\section{Metodología}

La investigación tuvo como objetivo estudiar el proceso de uso de sistemas remotos en el proceso de formación de estudiantes de instituciones de educación superior. Participaron en el estudio estudiantes de tres escuelas superiores de Rusia. Se realizó una encuesta electrónica para el estudio. El número total de encuestados fue de 542. De ellos, el $69 \%$ eran mujeres y el $31 \%$ eran hombres. La edad de los encuestados osciló entre los 18 y los 23 años (en su mayoría estudiantes a tiempo completo).

Se envió a los participantes una oferta para participar en la encuesta a través de un proveedor reconocido. Los que aceptaron la invitación fueron seleccionados mediante un conjunto de preguntas preestablecidas. Después de eso, solo aquellas personas que cumplen con las cuotas establecidas participaron en la encuesta principal. Este método de realización de la encuesta facilitó la recopilación rápida de datos y no requirió gastos importantes. Los encuestados respondieron ellos mismos a las preguntas (se excluyó la influencia del entrevistador). Para el estudio, se codificó el cuestionario y se formó una base de datos con las direcciones de correo electrónico de las personas que componen una amplia representación de la muestra objetivo.

Se estudió la experiencia de organizar la educación a distancia en 2020. Durante este tiempo, ha habido un fuerte salto en el uso de sistemas electrónicos como Zoom, Mirapolis, Team Viewer, Skype y otros. Se determinaron estadísticas sobre el uso de sistemas electrónicos para la realización de webinars y se estableció el número total de 
๔éduweb

Revista de Tecnología de Información y Comunicación en Educación • Volumen 15, Nㅜ 1. Enero-abril 2021

webinars realizados por instituciones de educación superior en disciplinas profesionales.

\section{Resultados y discusión}

La base del sistema de educación a distancia en las instituciones de educación superior es Moodle, que tiene una amplia gama de funciones y se puede utilizar de forma gratuita. La Sala Virtual de Mirapolis, Zoom y Time Viewer se utilizan en el proceso educativo de las instituciones de educación superior que estudiamos como sistemas auxiliares que brindan webinars. En el curso en Moodle, el docente cuenta con conferencias electrónicas, tareas, foros, etc. En este caso, los webinars se realizan en uno de los sistemas propuestos. La audiencia virtual reproduce el proceso de aprendizaje tradicional, en el que participa el hablante (puede ser un profesor o un alumno) y los oyentes participan en la discusión de un tema en particular.

Cada videoconferencia está encriptada y solo puede unirse a ella con una clave de acceso de un solo uso. Los profesores utilizan configuraciones adicionales (niveles de acceso de usuario). Las "salas de espera" se activan para que los participantes individuales de los webinars activen el proceso educativo. Se distribuyen los roles de los participantes. El profesor organiza a los estudiantes en grupos y, por lo tanto, rastrea la presencia de cada estudiante en la clase.

Todas las herramientas de gestión están en manos del gerente. Utiliza elementos interactivos para involucrar a los participantes. El docente puede nombrar a un coadministrador asistente, a quien se le otorgan las mismas capacidades para usar las herramientas (encender y apagar micrófonos, renombrar videochats y dividirlos en "salas", etc.) (Kidina, 2020). El profesor o su coadministrador permite que los participantes de la conferencia compartan pantallas de forma independiente.

Las funciones de Skype, WhatsApp y FaceTime se utilizan en el proceso de formación. Skype en un webinar realizado con la participación de 50 personas. El profesor ajusta los parámetros de audio y video de forma independiente. FaceTime se usa con menos frecuencia, porque solo pueden usarlo los propietarios del sistema iOS. Puede usar WhatsApp para realizar llamadas de voz en vivo, videollamadas, audio grupal y videoconferencias. Sin embargo, este servicio limita el número de participantes.

La experiencia de los webinars regulares en las universidades nos permite hablar de ellos como una herramienta productiva para que los estudiantes dominen materiales innovadores relevantes, ya que docentes y estudiantes de diferentes universidades de diferentes ciudades tienen la oportunidad de asistir a los webinars. El número de servicios utilizados es cada vez mayor y cada institución educativa tiene la oportunidad de elegir el más adecuado para fines específicos. 
La Figura 1 muestra una captura de pantalla de la pantalla durante una clase con estudiantes en Zoom.

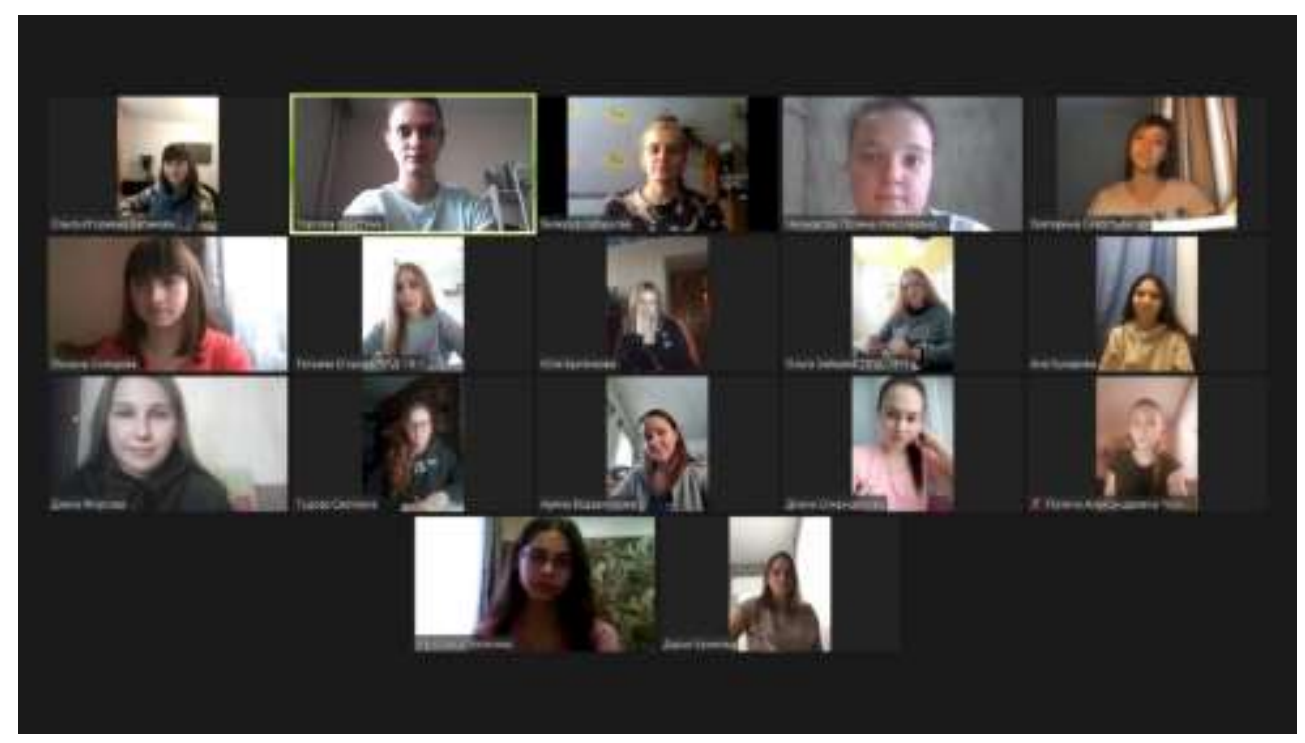

Fig. 1. Enseñando a los estudiantes en Zoom.

La Figura 2 muestra estadísticas sobre la realización de seminarios web en disciplinas profesionales durante tres años. En el aula, los estudiantes discuten temas profesionales y desarrollan estrategias con los maestros para lograr metas específicas.

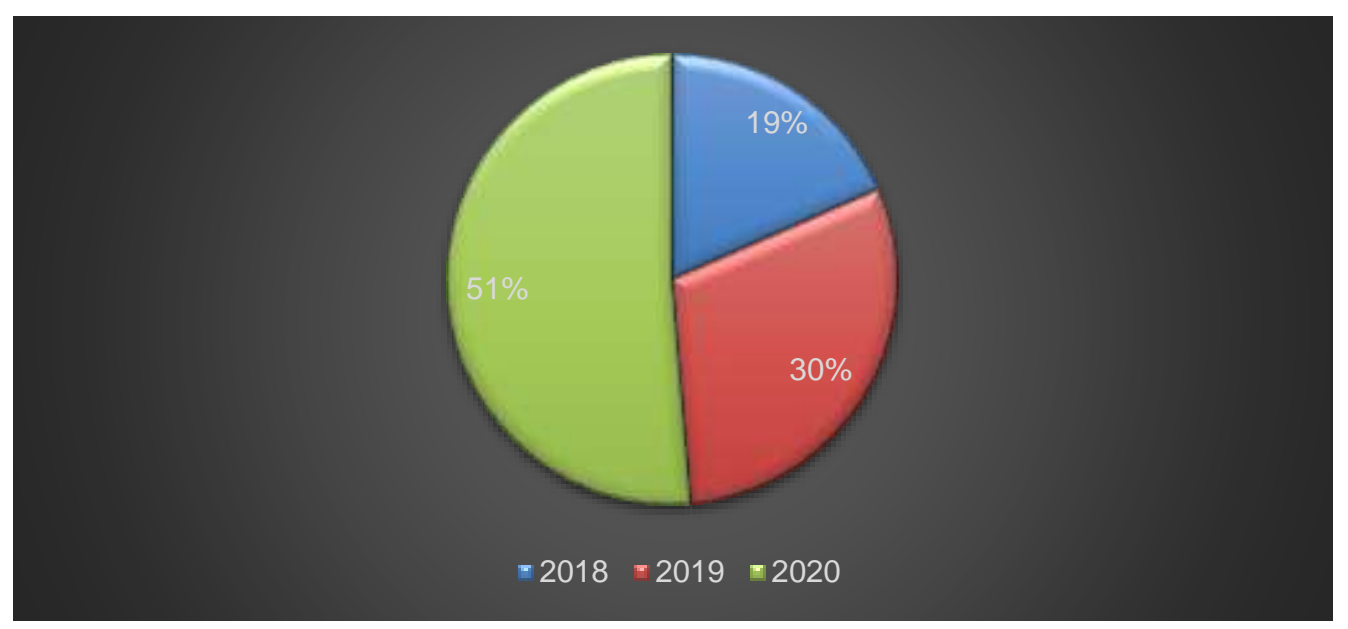

Fig. 2. El número de seminarios web realizados por instituciones de educación superior en disciplinas profesionales como porcentaje.

El número de webinars que realizan las instituciones de educación superior a clases en tiempo real se ha incrementado significativamente para 2020. En primer lugar, esto se debe al cumplimiento de los requisitos establecidos por la legislación sobre el 
desarrollo del entorno electrónico. Las escuelas superiores desarrollan cursos electrónicos y el personal docente realiza formación avanzada para trabajar en un entorno educativo moderno. Otro motivo global del incremento en el número de webinars es la transición al aprendizaje a distancia para preservar la salud de los sujetos del proceso educativo debido a la propagación de la infección por coronavirus. El aprendizaje remoto es una medida necesaria para mantener la distancia social.

La Figura 3 muestra estadísticas sobre el uso de sistemas electrónicos para seminarios web.

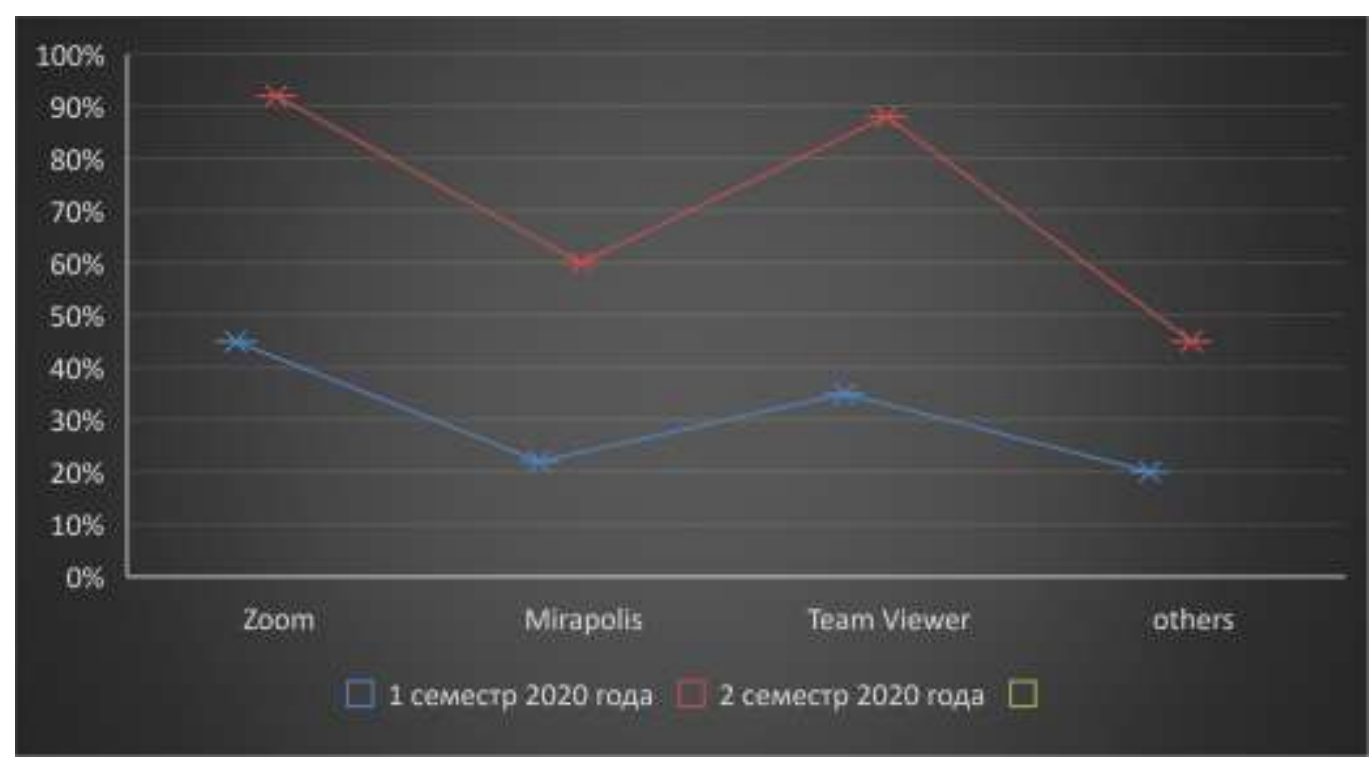

Fig. 3. Sistemas electrónicos utilizados por las escuelas superiores para realizar seminarios web (2020)

El semestre de invierno de 2020 se caracteriza por un fuerte salto al uso de varios sistemas electrónicos. Zoom es elegido por las instituciones de educación superior por muchas razones, incluido el funcionamiento estable del sistema a pesar de la carga creciente.

Encuestamos a estudiantes de instituciones de educación superior sobre las dificultades encontradas en el proceso de uso de sistemas remotos. Al comienzo del semestre, los estudiantes notaron que no podían usar dispositivos técnicos apropiados para participar en conferencias. Hasta la fecha, el porcentaje de estudiantes que no tienen esta oportunidad ha disminuido al $1 \%$ del número total de encuestados. Menos del $1 \%$ de los estudiantes con computadora o laptop no tienen conexión a Internet. Puede ver los materiales de formación desde cualquier dispositivo. Por un lado, aumenta la movilidad de los estudiantes y amplía sus oportunidades, pero por otro lado, como señalan los propios estudiantes, es perjudicial para la salud. El $25 \%$ de los encuestados dice que el uso de teléfonos inteligentes y tabletas es inconveniente en 
ausencia de comp utadoras portátiles y computadoras. Una diagonal de pantalla pequeña reduce la visión cuando se visualizan materiales de presentación con regularidad. Sin embargo, muchos participantes de la educación a distancia no tienen una sala separada para aprender. Hay un problema al mostrar el espacio de la casa. Zoom ofrece la posibilidad de utilizar un fondo virtual durante un chat de video. Los estudiantes eligen las opciones disponibles de "Fondo de pantalla" o cualquier otra imagen que será visible para todos los participantes de la videoconferencia en lugar del interior de la casa.

A pesar de la solución a muchos problemas que existían al comienzo de la transición masiva al aprendizaje en línea, el problema de reemplazar la comunicación real por la comunicación virtual sigue siendo relevante. El resultado del aprendizaje a distancia es una deformación cada vez mayor de la práctica de la interacción lingüística cara a cara entre los estudiantes. El $72 \%$ de los estudiantes está en contra de la transición masiva al aprendizaje en línea. No están satisfechos con el uso regular de sistemas remotos para impartir clases. Además, no todas las clases se pueden implementar mediante sistemas remotos. Para la implementación de capacitación de alta calidad, se revisó el contenido de las conferencias y los procedimientos de evaluación, y se corrigieron las tareas. Si en las etapas iniciales de uso de Zoom, Mirapolis, Team Viewer y otros sistemas hubo dificultades con el registro, el ingreso a la conferencia, la planificación de la conferencia, el uso de diversas funciones, tanto de profesores como de alumnos, hoy estos problemas de organización son insignificantes.

La Tabla 1 muestra las características comparativas de los sistemas de aprendizaje a distancia Zoom, Mirapolis y Time Viewer.

Tabla 1. Características comparativas de los sistemas de educación a distancia.

\begin{tabular}{lccc}
\hline Función & Zoom & Sala Virtual Mirapolis & TeamViewer \\
\hline Espacio para la colaboración & + & + & + \\
\hline Video & + & + & + \\
\hline $\begin{array}{l}\text { Demostración de archivo de } \\
\text { conferencia }\end{array}$ & - & + & + \\
\hline Compartir pantalla & + & + & - \\
\hline Chat en línea compartido & + & - & + \\
\hline Chat en línea personal & - & + & + \\
\hline Grabación de pantalla & - & + & + \\
\hline Gestión de roles de miembros & + & & + \\
\hline
\end{tabular}

La funcionalidad de cada uno de los sistemas presentados es bastante amplia. Cada uno de los sistemas presentados tiene el conjunto de funciones necesarias para realizar un webinar de capacitación. 


\section{Conclusiones}

La consideración de la experiencia de varios sistemas de educación a distancia muestra que su utilización por parte de las instituciones de educación superior para realizar seminarios web es de gran importancia para la organización de la formación moderna. Los webinars en condiciones modernas amplían las posibilidades de formación de estudiantes. En el proceso de transición masiva al proceso de aprendizaje a distancia, se descubrieron muchos problemas relacionados tanto con el aspecto técnico del problema como con el aspecto organizativo. Como mostró el estudio, se superaron muchas dificultades reestructurando las tareas, ajustando el contenido de las disciplinas y muchas otras modificaciones. Hoy, existe un agudo problema de aislamiento social y sustitución de la comunicación real de los estudiantes por la virtual. A pesar de brindar oportunidades para una interacción efectiva y asegurar la realización de las tareas, los sistemas electrónicos modernos no permiten satisfacer la necesidad de los sujetos del proceso educativo en la comunicación, lo cual es de gran importancia para los estudiantes.

La dinámica de implementación de Zoom, Mirapolis, Time Viewer y otros sistemas que estudiamos muestra la creciente demanda de sistemas de educación a distancia en la organización de capacitaciones y realización de webinars, entre otras cosas. Los procesos de ajuste realizados para mejorar la educación a distancia han permitido eliminar muchos errores y carencias, y realizar cambios en el proceso educativo. Pero a pesar de esto, la implementación de las capacidades de los sistemas remotos requiere más estudio y corrección.

\section{Referencias}

Chil Núñez, I., Escalona Arranz, J., Molina Bertrán, S., Dutok Sánchez, C., Arias Ramos, D., Pérez Rondón, L., Ochoa Pacheco, A., \& Picanço Souto, R. (2020). Perfeccionamiento de la estrategia curricular de medio ambiente de la carrera de ciencias farmacéuticas de la Universidad de Oriente, Cuba. Revista Científica Del Amazonas, 3(5), 6-17. https://doi.org/10.34069/RC/2020.5.01

Decree of the President of the Russian Federation No. 204. On the national and strategic objectives of the development of the Russian Federation until 2024, dated 07.05. 2018

Eliseeva, D.Yu., Fedosov, A.Yu., Agaltsova, D.V., Mnatsakanyan, O.L., \& Kuchmezov, Kh.Kh. (2020). The evolution of artificial intelligence and the possibility of its application in cyber games. Amazonia Investiga, 9(28), 123-129. https://amazoniainvestiga.info/index.php/amazonia/article/view/1043

Kidina, L.M. (2020). Management of the pedagogical collective in the conditions of implementing distance learning. Baltic Humanitarian Journal, 9 No 4 (33), 93-96.

Nagovitsyn, R.S., Vaganova, O.I., Kutepov, M.M., Martyanova, L.N., Kosenovich, O.V., Moeseev, Yu.V., Vorotova, M.S., \& Osipov, A.Y. (2020). Interactive Technologies In Developing Student's Motivation In Physical 
Education And Sport. International Journal of Applied Exercise Physiology, 9(6), 78-85.

Nikonova, N.P., Vaganova, O.I., Smirnova, Zh.V., Chelnokova, E.A., Kutepov, M.M. (2019b). Methodological support in partnerships with the institution of additional education and teachers. International Journal of Applied Exercise Physiology, 8(2.1), 382-390.

Nikonova, N.P., Vaganova, O.I., Smirnova, Zh.V., Bystrova, N.V., \& Markova, S.M. (2019a). Providing partnerships and promotion of additional educational services. International Journal of Applied Exercise Physiology, 7(2.1), 391-399.

Pichugina, G.A., \& Bondarchuk, A.I. (2019). Structure of the training case in the organization of the educational process. Humanitarian Balkan Research, 2(4), 5-7.

Ponachugin, A.V (2020). Practice of using interactive technologies for bachelor of applied computer science training during the Covid-19 Pandemic. Vestnik of Minin University, 8(4), 6. (In Russ)

Sarmiento Orna, D., \& Vinueza Martínez, J. (2020). Percepción estudiantil de la calidad del servicio universitario: caso de una Universidad Ecuatoriana. Revista Científica Del Amazonas, 3(5), 54-66. https://doi.org/10.34069/RC/2020.5

Shcerbakova, E.V., \& Shcerbakova, T.N. (2019). Experience of use of remote computer technologies at the organization of independent work of students in the conditions of a mark and rating system. Baltic Humanitarian Journal, 8, No 4 (29), 192-195.

Vaganova, O., Livshits, Y., Aleshugina, E., Smirnova, Z., \& Kutepova, L. (2019a). Experience in Developing Electronic Glossary in A Higher Education Institution. Amazonia Investiga, 8(22), 247-253. Retrieved from Https://Amazoniainvestiga.Info/Index.Php/Amazonia/Article/View/421

Vaganova, O., Rudenko, I., Markova, S., Smirnova, Z., \& Kutepov, M. (2019b). The use of educational video materials in educational process of a higher educational institution. Amazonia Investiga, 8(22), 216-222. Retrieved from https://amazoniainvestiga.info/index.php/amazonia/article/view/308

Vaganova, O.I., Petrozitskaya, I.A., Snatovich, A.B., Odarich, I.N., \& Kirillova, I.K. (2020). Heuristic technologies of training in professional education. Amazonia Investiga, $9(27)$,

509-517.

https://amazoniainvestiga.info/index.php/amazonia/article/view/1265

Vaganova, O.I., Bakharev, N.P., Bulaeva, M.N., Lapshova, A.V., \& Kutepov, M.M. (2020b). Methods and Means of realization of interaction technologies in the University. Amazonia Investiga, 9(26), 383-390. https://amazoniainvestiga.info/index.php/amazonia/article/view/1168

Vaganova, O.I., Bakharev, N.P., Kulagina, J.A., Lapshova, A.V., \& Kirillova, I.K. (2020c). Multimedia technologies in vocational education. Amazonia Investiga, $9(26)$, 391-398.

https://amazoniainvestiga.info/index.php/amazonia/article/view/1169 